\title{
Prevention of Dabigatran-related Gastrointestinal Bleeding With \\ Gastroprotective Agents: A Population-Based Study
}

Esther W Chan*, ${ }^{1}$ Wallis CY Lau*, ${ }^{1}$ Wai K Leung, ${ }^{2}$ Michael TC Mok, ${ }^{3}$ Ying He, ${ }^{1}$ Teresa SM Tong, ${ }^{2}$ Ian CK Wong ${ }^{1}$

*Co-first author

${ }^{1}$ Centre for Safe Medication Practice and Research, Department of Pharmacology and Pharmacy, Li Ka Shing Faculty of Medicine, The University of Hong Kong; ${ }^{2}$ Department of Medicine, Li Ka Shing Faculty of Medicine, The University of Hong Kong; ${ }^{3}$ Department of Cardiology, Geelong Hospital and Deakin University, Victoria, Australia.

Corresponding Author: Professor Ian CK Wong, Centre for Safe Medication Practice and Research, Department of Pharmacology and Pharmacy, Li Ka Shing Faculty of Medicine, The University of Hong Kong, L02-56, 2/F, 21 Sassoon Road, Li Ka Shing Faculty of Medicine, Laboratory Block, Faculty of Medicine Building, Hong Kong SAR, China (Tel: (852) 3917 9250;

Fax: (852) 2817 0859; Email: wongick@hku.hk).

Funding support: This study was supported by the General Research Fund, Research Grants Council, Hong Kong (project number: 17102314).

Conflict of Interest Disclosures: Dr. Chan has received research support from Bristol-Myers Squibb; and Janssen, a division of Johnson and Johnson. Prof. Leung has received speaker fees from Eisai, Ferring, Menarini and Takeda. He also serves on the advisory board of AbbVie, Boehringer-Ingelheim and Janssen. The remaining authors disclose no conflicts. 
Author Contributions: Dr. Chan and Ms. Lau had full access to all of the data in the study and take responsibility for the integrity of the data and the accuracy of the data analysis.

Study concept and design: Chan, Lau, Wong.

Acquisition, analysis, or interpretation of data: Chan, Lau, Wong, Leung, Mok, He, Tong.

Drafting of the manuscript: Chan, Lau.

Critical revision of the manuscript for important intellectual content: Chan, Lau, Leung, Wong.

Statistical analysis: Lau, He.

Obtained funding: Chan, Leung, Mok, Wong.

Administrative, technical, or material support: He, Tong.

Study supervision: Wong

Abbreviations used in this paper: GIB (gastrointestinal bleeding), PPIs (proton pump inhibitors), H2RAs (histamine type-2 receptor antagonist), GPA (gastroprotective agents), IRR (incidence rate ratio), CI (confidence intervals), NOACs (novel oral anticoagulants), AF (atrial fibrillation), RE-LY (Randomized Evaluation of the Long-Term Anticoagulation Therapy), HA (Hospital Authority), CMS (Clinical Management System), CDARS (Hong Kong Clinical Data Analysis and Reporting System), PPV (positive predictive value), NPV (negative predictive value), HR (hazard ratio), NSAIDs (nonsteroidal anti-inflammatory drugs), SSRIs (selective serotonin reuptake inhibitors), TIA (transient ischemic attack), SE (systemic embolism), VTE (venous thromboembolism).

\section{Word count: 5,947}

No. of Tables: 4 
No. of Figures: 2

No. of Supplementary Table: 1

No. of Supplementary Figure: 1 


\begin{abstract}
BACKGROUND\&AIMS: Use of dabigatran, an inhibitor of thrombin, increases risk of gastrointestinal bleeding (GIB). However, it is not clear whether gastroprotective agents (GPAs) prevent GIB in dabigatran users. We investigated the risk of GIB and the role of GPAs (including proton-pump inhibitors and histamine type-2 receptor antagonists) in patients using dabigatran.
\end{abstract}

METHODS: We performed a retrospective cohort study using a population-wide database managed by the Hong Kong Hospital Authority. Patients newly prescribed dabigatran from 2010 through 2013 were included in the analysis. Poisson regression was used to assess the risk of GIB in dabigatran users by incidence rate ratio (IRR), adjusted for patient characteristics, comorbidities, and concurrent medications.

RESULTS: Among the 5041 patients newly prescribed dabigatran, 124 (2.5\%) developed GIB during follow-up (4.2/100 patient-years). The risk of GIB in this population increased among patients 75 y old or older (IRR, 2.47; 95\% confidence interval [CI], 1.66-3.68), patients with a history of peptic ulcers or GIB (IRR, 2.31; 95\% CI, 1.54-3.46), and patients who used aspirin (IRR, 1.52; 95\% CI, 1.03-2.24). Concomitant use of GPAs was associated with a reduced risk of GIB (IRR, 0.52; 95\% CI, 0.35-0.77). Subcategory analysis showed that use of proton-pump inhibitors (IRR, 0.53 ; 95\% CI, 0.31-0.91) or histamine type-2 receptor antagonists (IRR, 0.61 ; 95\% CI, 0.40-0.94) were associated with lower risk of GIB. Further analysis revealed that the risk reduction by GPAs was significant for only upper GIB (IRR, 0.29; 95\% CI, 0.15-0.54) and only for patients with prior history of peptic ulcers or GIB (IRR, 0.14; 95\% CI, 0.06-0.30). 
CONCLUSIONS: In the Hong Kong population, use of GPAs and was associated with a reduced risk of GIB in patients taking dabigatran. The association was stronger for upper GIB than lower GIB, and in patients with prior history of peptic ulcers or GIB.

KEYWORDS: anticoagulant; drug side effect; PPI; H2RA

Word count: 312 


\section{Introduction}

Dabigatran, an oral direct thrombin inhibitor ${ }^{1}$, is the first novel oral anticoagulants (NOACs) approved for the prevention of stroke and systemic embolism in patients with non-valvular atrial fibrillation (AF). Unlike traditional oral anticoagulant warfarin, dabigatran has a predictable pharmacokinetic profile and does not require frequent blood monitoring. In the Randomized Evaluation of the Long-Term Anticoagulation Therapy (RE-LY) trial, dabigatran 150mg twice daily was superior to warfarin in the prevention of stroke and systemic embolism in AF patients and non-inferior to warfarin at $110 \mathrm{mg}$ twice daily. ${ }^{2}$

Despite its comparable efficacy and relative convenience, several randomized controlled trials and reports have demonstrated that the risk of gastrointestinal bleeding (GIB) is higher with the use of dabigatran than traditional therapies across different indications. ${ }^{2-5}$ A recent systematic review on previous clinical trials demonstrated that the pooled odds ratio (OR) of GIB associated with dabigatran use was 1.6 when compared to traditional therapies including warfarin. ${ }^{6}$ Recently, the U.S. Food and Drug Administration (FDA) released a safety announcement that dabigatran is associated with a higher rate of gastrointestinal bleeding compared with warfarin in patients with non-valvular AF, based on their latest analysis of the Mini-Sentinel database. ${ }^{7}$ However, the actual bleeding risk of dabigatran use for various indications in daily clinical practice outside restrictive clinical trial setting is less well described.

Although current guidelines recommend that proton pump inhibitors (PPIs) should be considered in patients at high risk of GIB receiving antithrombotic therapy, ${ }^{6,8-10}$ the role of gastroprotective agents including PPIs and histamine type-2 receptor antagonists (H2RAs) in the prevention of GIB associated with dabigatran remains undefined. 
This study determined the risk of GIB in patients newly prescribed with dabigatran in a large population based retrospective cohort study from Hong Kong. We also identified the risk factors and the effects of gastroprotective agents in dabigatran associated GIB in this cohort.

\section{Method}

\section{Data source}

This study used the electronic medical records of the Clinical Data Analysis and Reporting System (CDARS) of the Hong Kong Hospital Authority (HA), which is the sole public-funded healthcare provider of Hong Kong. The HA is currently serving a population of over seven million, managing 42 hospitals, 47 Specialist Out-patient Clinics, and 73 General Out-patient Clinics organized into seven hospital clusters according to geographical locations. ${ }^{11}$ The HA uses the Clinical Management System (CMS), a computerized clinical management system, to record all key clinical information such as treatment, diagnoses, prescriptions, laboratory results and procedures information; and to write consultation and discharge summaries. ${ }^{12}$ It also allows clinicians and health care specialists to order and review care in their daily practice. ${ }^{13}$ Electronic patient records in the HA, including demographics, date of consultation, date of hospital admission and discharge, diagnoses, procedures, drug prescriptions and laboratory tests were transferred from CMS to CDARS for audit and research propose. ${ }^{13}$ CDARS has been used for conducting high quality population-based studies. ${ }^{13-17}$ A local study demonstrated a high accuracy of coding in CDARS with a positive predictive value of $90 \%{ }^{18}$ Nonetheless, we conducted further validation on a sample of patients in this cohort to ensure the validity of our dataset. 
All patient records in CDARS are anonymized to protect patient confidentially. A unique reference number is allocated for each individual patient to facilitate data retrieval and further

analysis. The study protocol was approved by the Institutional Review Board of the University of Hong Kong/Hospital Authority Hong Kong West Cluster (reference number: UW 13-468). Informed consent from patients was not required since the data used in this study were anonymized.

\section{Study design}

This is a retrospective cohort study. We identified all patients who were newly prescribed with dabigatran between $1^{\text {st }}$ Jan 2010 and $31^{\text {st }}$ Dec 2013 from the CDARS. The index date was defined as the date of the first dabigatran prescription. The follow-up of each patient was commenced from the index date until the development of GIB, death, the prescription of an alternative anticoagulant (warfarin, rivaroxaban or apixaban), the end of the study period ( $31^{\text {st }}$ Dec 2013) or 30 days after discontinuation (defined as $>30$ days of interval between prescription refills) of dabigatran, whichever came first. Another 30-day follow-up was added after the final prescription of dabigatran to account for non-compliance and capture any GIB that may have led to treatment discontinuation. Patients who had received dabigatran in the 12 months prior to the index date and patients who were prescribed other anticoagulants on the index date were excluded. Patient's coexisting medical illnesses prior to the index date and drugs concurrently prescribed in the follow-up period were retrieved from the CDARS (Figure 1).

\section{Outcome and covariates}

The primary outcome was the development of GIB after the prescription of dabigatran. Patients who developed GIB were identified in CDARS with the physician assigned International 
Classification of Diseases codes (Ninth Revision, Clinical Modification (ICD-9); Supplementary Table 1). We included possible diagnoses of GIB including peptic ulcer hemorrhage (531.0, 531.2, 531.4, 531.6, 532.0, 532.2, 532.4, 532.6, 533.0, 533.2, 533.4, 533.6, 534.0, 534.2, 534.4, 534.6), bleeding gastritis and/or duodenitis (535.01, 535.11, 535.21, 535.31, 535.41, 535.51, 535.61, 535.71), intestinal hemorrhage $(562.02,562.03,562.12,562.13,569.3,569.85,569.86)$ and gastrointestinal hemorrhage (578). Upper GIB and non-upper GIB were classified by the diagnostic codes and free text information in CDARS (Supplementary Table 1).

The use of the following medications during follow-up was included as covariates in terms of dichotomous variables (present/absent) in the analysis (Figure 1): use of aspirin, clopidogrel, nonsteroidal anti-inflammatory drugs (NSAIDs), proton pump inhibitors (PPIs), histamine type-2 receptor antagonists (H2RAs), and selective serotonin reuptake inhibitors (SSRIs). ${ }^{19-23}$ Baseline medical conditions including prior ischemic stroke/transient ischemic attack/systemic embolism, renal disease, and prior history of peptic ulcer/GIB were also counted as dichotomous covariates in the analysis. ${ }^{20-22}$ For each patient, all diagnosis records dated prior to the individual index date were retrieved using ICD-9 codes (Supplementary Table 1) from CDARS for the identification of the baseline medical conditions.

\section{Data validation}

To validate the coding accuracy, we extracted the original medical records in the CMS of a sample of patients (Supplementary Figure 1) from the Hong Kong West (HKW) cluster, which is one of the seven hospital clusters (a group of hospitals under the same management structure in a region of Hong Kong) of the HA. The HKW cluster comprises seven hospitals, one specialist rehabilitation center, and six general out-patient clinics in the Central \& Western District and the 
Southern District of Hong Kong. ${ }^{24}$ The HKW cluster region has a population of over half a million, representing about $8 \%$ of the Hong Kong population. ${ }^{24}$ The age and gender distribution in the HKW cluster region are similar to that of the overall population in Hong Kong. ${ }^{25}$ The HKW cluster also provides services to patients residing outside its cluster region through crosscluster referrals. In particular, it hosts one of the two university teaching hospitals in Hong Kong, the Queen Mary Hospital, which is a tertiary referral center for all complex cases. ${ }^{24}$

A gastroenterologist (WKL) manually reviewed the patient records to validate the diagnosis of GIB (Supplementary Table 1). The identifications of two important patient characteristics were also validated in our cohort from CDARS - prior history of peptic ulcer/GIB and history of AF (as indication). The cases of GIB and peptic ulcers were confirmed by endoscopy findings, pathology reports, surgery, radiology, autopsy, clinical symptoms (e.g. hematemesis, melena) or documentation of the diseases in medical chart. The diagnosis of AF was ascertained by

electrocardiogram, Holter monitor findings, or any documentation of the disease history or indication for dabigatran. Patients on dabigatran with no GIB, no prior history of peptic ulcer/GIB, or received dabigatran for indications other than AF were randomly selected to serve as negative controls. The corresponding positive predictive value (PPV) and negative predictive value (NPV) were calculated.

\section{Statistical analysis}

Continuous variables were reported as mean \pm standard deviation whereas categorical data were expressed as frequencies (percentages), respectively. Risk time was measured from the index date when dabigatran was prescribed until the development of GIB. Conditional Poisson regression was used to determine the risk of GIB, in terms of incidence rate ratio (IRR) with 95\% 
confidence intervals (CI), among patients who were taking dabigatran after controlling for baseline medical conditions and use of concurrent medications. Patients who had a prescription of either H2RA and/or PPI during follow-up (regardless of the duration of medication) were defined as users of gastroprotective agents. Subgroup analyses were conducted by classifying patients into three groups according to their drug exposures: 1) H2RA only; 2) PPI only; and 3) both H2RA and PPI (either prescribed simultaneously or sequentially).

The times to GIB for users and non-users of gastroprotective agents were illustrated by KaplanMeier curves and compared using the Cox proportional hazards regression model in hazard ratios (HR), with adjustment for baseline characteristics and use of concurrent medications as previously mentioned.

We conducted further subgroup analysis to examine the effect of gastroprotective agents in new OAC "starters" and "switchers" from other OACs, since they may have different bleeding risks. ${ }^{26}$ A patient was classified as a "switcher" if they were prescribed warfarin, rivaroxaban or apixaban within 3 months prior to index date. We also performed subcategory analyses to assess the effects of gastroprotective agents for upper GIB and non-upper GIB. Since a number of case reports revealed an early onset of dabigatran-associated GIB, ${ }^{27-30}$ we conducted an additional analysis to evaluate the protective effect of gastroprotective agents on early bleeding defined as occurring within 30 days since commencement of dabigatran. For this analysis, only the first 30 days since dabigatran commencement were included.

A two-sided p-value <0.05 was considered as statistically significant. Statistical Analysis System (SAS) v9.3 (SAS Institute Inc., Cary, North Carolina) was used for statistical analysis. 


\section{Sensitivity analysis}

Several additional analyses were conducted to test the robustness of the main results. Since oral anticoagulants could exacerbate bleeding from underlying lesions, patients with prior history of peptic ulcer/GIB may be at higher risk of GIB. ${ }^{6,22}$ Therefore, in the first sensitivity analysis, we stratified the patients by prior history of peptic ulcer/GIB to explore the effect of gastroprotective agents in different risk groups. Another analysis was conducted by inclusion of AF patients only, which is the most common indication for dabigatran. The third sensitivity analysis accounted for the dosage effect of dabigatran on GIB by including the average daily dose as a covariate in an additional analysis. Finally, additional analyses were performed by stratifying the users of gastroprotective agents into patients who were continuously prescribed gastroprotective agents (continuous users), defined as those with a recorded supply of medication for $\geq 80 \%$ of the days of follow-up; and those who were not continuous users (i.e. infrequent users).

\section{Results}

\section{Data validation}

Among the 5,041 patients on dabigatran included in this study, $711(14.1 \%)$ patients were from the HKW cluster (Supplementary Figure 1). Of these, we selected all patients who had a diagnosis of GIB $(n=35)$, all patients who had prior history of peptic ulcer/GIB $(n=78)$, and a random sample of $133(20 \%)$ patients who had AF. As negative controls, we selected a random sample of $66(10 \%)$ patients who did not develop GIB, a random sample of $63(10 \%)$ patients who did not have prior history of peptic ulcer/GIB, and all patients who did not have AF (n=32). There were 63 patients being selected for validation of two medical conditions, and 8 patients being selected for validation of three medical conditions due to the random nature of the sample 
selection. The final validation sample consisted of 328 patients. The corresponding PPVs and NPVs were: GIB, PPV=100\%, NPV=98\%; prior history of peptic ulcer/GIB, PPV=90\%, $\mathrm{NPV}=92 \% ; \mathrm{AF}, \mathrm{PPV}=95 \%, \mathrm{NPV}=91 \%$.

\section{Patient characteristics}

In total, 5,206 patients received dabigatran during the study period. Of these, five patients had dabigatran prescription(s) within one year prior to index date and were excluded. Further, 160 patients who were being prescribed other oral anticoagulants on the index date were removed. The final analysis included 5,041 patients. The most common indication for dabigatran was AF (88.3\%). The mean age of the patients was $72.0 \pm 10.9$ years and $47.8 \%$ were male. The mean follow-up was $215 \pm 255$ days and the total follow-up was 2,973 patient-years. Baseline characteristics of the patients are presented in Table 1.

\section{Risk of gastrointestinal bleeding}

There were 124 patients (2.5\%) who developed GIB during follow-up. All cases were hospitalized with a diagnosis of GIB. Of these, 93 (75\%) patients were admitted to hospital with GIB, and the remaining $31(25 \%)$ patients developed GIB during hospitalization. The median length of hospital stay was 3 days (interquartile range: 5 days). Of the 124 bleeding cases, 55 (44.4\%) patients underwent endoscopy, 11 (8.9\%) patients received transfusion, and 7 (5.6\%) patients received both endoscopy and transfusion. There was one patient who died in the bleeding episode due to aspiration pneumonia. The median time to GIB was 36.5 days (interquartile range: 135.5 days) after the first prescription of dabigatran. The overall incidence of GIB in this cohort was 4.2 per 100 patient-years. 
Patients aged $\geq 75$ years (IRR, 2.47; 95\%CI, 1.66-3.68), with a history of peptic ulcer/GIB (IRR, 2.31; 95\%CI, 1.54-3.46), or with concomitant use of aspirin (IRR, 1.52; 95\%CI, 1.03-2.24) were found to have higher risk of GIB (Table 2).

The risk of GIB was however significantly reduced with the concomitant use of gastroprotective agents. After adjusting for baseline medical conditions and concurrent medications, a reduction of $48 \%$ in the risk of GIB was found in users of gastroprotective agent as compared with nonusers (IRR, 0.52; 95\%CI, 0.35-0.77) (Table 2). Sub-analysis showed that both PPIs (IRR, 0.53; 95\%CI, 0.31-0.91) and H2RAs (IRR, 0.61; 95\%CI, 0.40-0.94) significantly reduced the risk of GIB. A further risk reduction was found in patients utilizing both H2RAs and PPIs over time (IRR, 0.15; 95\%CI, 0.06-0.39). Figure 2 presents the Kaplan-Meier curves of the proportions of patients who were free of GIB. Similar risk reduction was noted among users of gastroprotective agents as compared with non-users (HR, 0.57; 95\%CI, 0.38-0.85).

Further analysis showed that the use of gastroprotective agents significantly reduced the risk of GIB in patients who had prior history of peptic ulcers/GIB (IRR, 0.14; 95\%CI, 0.06-0.30) but not in patients with no prior history (IRR, 0.80; 95\%CI, 0.50-1.28; Table 3). In the subcategory analyses of sources of GIB into upper GIB and non-upper GIB, gastroprotective agents were found to significantly reduce the risk of upper GIB (IRR, 0.29; 95\%CI, 0.15-0.54) but not nonupper GIB (IRR, 0.75; 95\%CI, 0.45-1.27).

The sensitivity analysis which included patients with AF only yielded similar results. The use of gastroprotective agents was associated with a reduced risk of GIB in AF patients (IRR, 0.58; 95\%CI, 0.39-0.87); (Table 3). Similarly, a lower risk of GIB was observed in both new OAC starters (IRR, 0.56; 95\%CI, 0.35-0.89) and switchers from other OAC (IRR, 0.38; 95\%CI, 0.18- 
0.82) receiving gastroprotective agents. Even after adjusting for different dosages of dabigatran, the effects of gastroprotective agents in preventing GIB remained significant (IRR, 0.53; 95\%CI, $0.36-0.80$ ). In the analysis for early GIB that developed within 30 days of dabigatran use, the concurrent use of gastroprotective agents were associated with a significant reduction in the risk of bleeding (IRR, 0.39; 95\%CI, 0.22-0.69).

Among all users of gastroprotective agents, about $70 \%$ used the drugs continuously during follow-up (Table 4). A reduction of the risk of GIB was observed among these patients as estimated by IRR $(0.51 ; 95 \% \mathrm{CI}, 0.34-0.77)$ and HR $(0.57 ; 95 \% \mathrm{CI}, 0.38-0.87)$. The effects of gastroprotective agents among the infrequent users were not statistically significant in general (Table 4).

\section{Discussion}

Gastrointestinal bleeding is a major adverse clinical outcome associated with the use of the NOACs. In particular, the bleeding risk appears to be higher for dabigatran when compared to other NOACs. ${ }^{6}$ As there is currently no effective antidote for dabigatran, patients are potentially at a higher risk of uncontrolled GIB as compared with warfarin, which can be reversed by vitamin $\mathrm{K}$ and fresh frozen plasma. This would highlight the importance of identifying a preventive strategy for GIB in patients taking dabigatran. This is the first population-based study that determines the role of gastroprotective agents on the risk of GIB among patients newly prescribed with dabigatran. We used the central electronic patient record database of the HA of Hong Kong which covers about $80 \%$ of all hospital admissions in Hong Kong. ${ }^{31}$ We found that the concomitant use of gastroprotective agents, including PPIs and H2RAs, significantly reduced the risk of GIB by $48 \%$ among patients on dabigatran. Of particular importance is that only 
patients with a history of peptic ulcers or GIB, who were considered a high-risk group for GIB, were found to be significantly protected by gastroprotective agents. While NOAC could result in bleeding from small bowel or lower GI tract, our sub-analysis showed that the protective effect was statistically significant for upper GIB only. The results were also consistent for all sensitivity analyses, including testing on different indications and dosage effects of dabigatran and prior OAC experience.

In this study, we found that old age (age $\geq 75$ ), a history of peptic ulcer/GIB and the concomitant use of aspirin were associated with higher risk of GIB among dabigatran users. This is consistent with previous observations that age and prior history of peptic ulcer/GIB are associated with higher risk of GIB among patients using NSAIDs or aspirin. ${ }^{32-34}$ Since many AF patients would have concurrent ischemic heart disease that would require aspirin, our data are supportive of further increase in risk of GIB when aspirin and dabigatran is co-administered. Particular caution is therefore mandatory in this group of patients who require both antiplatelet and anticoagulant therapies.

Notably, we found that $67.5 \%$ of the dabigatran users in this cohort were concurrently prescribed gastroprotective agents. While a similar prescription rate was observed in the AF patients in Italy $(65.3 \%)^{35}$, the prescription rate is higher than those reported in the RE-LY trial $(17.6 \%)^{2}$ and other observation studies in the United States $(24 \%)^{36}$ and Denmark $(13.4 \%)^{37}$. A plausible explanation of the high prescribing rate is that clinicians may be more cautious about peptic ulcer disease and bleeding since gastrointestinal disorders is one of the most common diseases requiring hospital admission in Hong Kong. ${ }^{31}$ When compared to the West, Hong Kong has a higher prevalence of peptic ulcer disease. ${ }^{38-40}$ Therefore, it is possible that clinicians in Hong Kong have a higher tendency of prescribing gastroprotective agents compared to other countries. 
Further, medications in HA are highly subsidized ( $85 \%$ to $98 \%$ ) by the government. ${ }^{41}$ The low price of gastroprotection medications might also favor its use for various indications such as prophylaxis of ulcer and bleeding, maintenance therapy for gastroesophageal reflux diseases and non-cardiac chest pain among dabigatran users.

The exact mechanism how gastroprotective agents prevent upper GIB in patients taking dabigatran remains unknown. Whilst the median time to bleeding after the use of dabigatran was approximately one month, the co-administration of PPI or H2RA may reduce the risk of bleeding from pre-existing gastroduodenal ulcers or erosions. Hence, the risk reduction was significantly higher among patients with prior history of peptic ulcer disease/GIB. Another possible explanation for the observed protective effect of gastroprotective agents may be mediated through acid suppression that interferes with absorption of dabigatran. The formulation of dabigatran etexilate contains a tartaric acid core to provide an acidic environment required for drug absorption. ${ }^{1,2}$ It is biologically plausible that the acid suppressive effects of PPIs and H2RAs reduce the absorption of dabigatran. In the RE-LY trial, PPIs were observed to lower dabigatran exposures by $15 \%$, but did not significantly reduce the overall clinical efficacy. ${ }^{42}$ Further study is warranted to assess the potential risk-benefits of gastroprotection in patients on dabigatran by quantifying the risk of thromboembolism versus the benefit of preventing GIB.

Unlike previous randomized controlled studies that had restrictive patient's selection criteria, ${ }^{2-4}$ our results were based on real-life clinical practice, through inclusion of all patients who were prescribed dabigatran. We utilized electronic database resources, which have been recognized to provide powerful platforms to conduct large-scale, real-life drug safety and post-marketing surveillance studies. ${ }^{43-46}$ By using the largest inter-linked clinical database in Hong Kong, we 
observed a higher risk of dabigatan-related GIB in the Southern Chinese population group (4.2 per 100 person-year) compared to other non-Asians patient groups as reported in Denmark (1.2 to 1.5 per 100 person-year $)^{37}$ and in the United States (0.6 to 3.4 per 100 person-year). ${ }^{7,47}$ The higher bleeding risk observed in our study population could not be explained by the dose of dabigatran used, as most (73\%) of our patients were using the lower dosage of $220 \mathrm{mg} / \mathrm{day}$. In the RE-LY trial, the bleeding risk of Asians was higher than that of non-Asians despite younger age and comparable blood pressure. ${ }^{48}$ Similarly findings were reported in a retrospective cohort study of 18,867 AF patients that Asians had a higher risk of intracranial hemorrhage compared with the Whites, Blacks, and Hispanics. ${ }^{49}$ A possible mechanistic explanation of this observation could be explained by the genetic differences in blood coagulation between Asians and non-Asians. ${ }^{48}$ In particular, the factor $\mathrm{V}$-Leiden mutations, which favors the formation of blood clots, is extremely rare in Asian but more commonly found in other ethnicities. ${ }^{50,51}$ It remains to be determined whether there are considerable ethnic differences on the clearance of drugs or whether the Chinese population is more susceptible to bleeding. ${ }^{52,53}$

Our study has limitations. Similar to other clinical healthcare databases, CDARS does not capture the use of over-the-counter medications such as NSAIDs, which could lead to underestimation of the effect of concurrent medications on bleeding risk. However, in Hong Kong, non-aspirin oral NSAIDs in general are not over-the-counter medications and require a doctor's prescription. ${ }^{54}$ Furthermore, the HA is the only publicly-funded primary care in Hong Kong where services and medications are highly subsidized (85\% to $98 \%$ ) by the government. ${ }^{41}$ As a result, it is common for patients with chronic illness requiring long-term medications, such as patients with AF on dabigatran, to attend outpatient clinics of HA for ongoing treatment care rather than obtaining full-cost medications from over-the-counters. ${ }^{41}$ Therefore, it is anticipated 
that the impact of missing of over-the-counter prescriptions would be minimal. As inherent in the retrospective cohort study design based on large population databases, we cannot exclude the possibility of residual confounding factors. In particular, other risk factors for peptic ulcer and bleeding such as Helicobacter pylori status and smoking history were lacking in this database. To overcome this potential limitation, we have included other possible confounding factors in our Poisson regression and added different sensitivity analyses in this study. The protective effects of PPI or H2RA appeared to be very consistent even after adjustment with indications or dosages of dabigatran and concurrent medical illnesses. With further analysis, the protective effect of gastroprotective agents against GIB was found to be more prominent in patients with prior history of peptic ulcer/GIB. Different sensitivity analyses or sub-analyses supported the consistency of the protective effects of gastroprotective agents.

Our study also did not include control groups or comparator arms including conventional oral anticoagulants such as warfarin. However, several published epidemiological studies and clinical trials have compared dabigatran with traditional therapies, which consistently infer that dabigatran has a comparable or higher risk of GIB when compared to warfarin..$^{2-4,7,37,55-57}$ Hence, our focus was to characterize the risks and protective factors of GIB associated with dabigatran, particularly in the Chinese population, where similar data are currently lacking. In addition, we could only access the medical records in CMS in a single hospital cluster for data validation, but not in all the seven clusters due to the limited access right. Therefore, potential bias could arise from any systematic differences in coding validity across different clusters. Nevertheless, CMS and CDARS are clinical health record databases. The information stored in CMS and CDARS are used for daily clinical management rather than insurance and reimbursement. Hence, the coding is not linked with any financial rewards or incentives, and the coding practice is most 
likely to be non-differential between individual patients and hospital clusters, which are under the same management structure of the HA. As in our validation sample, the bleeding rate (35/711 $=4.9 \%)$ appeared to be higher compared to the overall rate $(2.5 \%)$. Since the HKW cluster is a tertiary referral center which tends to have referrals from cross-cluster patients with complex medical problems, the inclusion of these patients may have higher risk of bleeding than other

patients. ${ }^{24}$ Finally, with a relatively smaller number of infrequent users of gastroprotective agents in this cohort, we cannot exclude the possibility that the non-significant results for the infrequent users were due to insufficient power. Further study is needed to confirm the results.

\section{Conclusions}

This study showed that the risk factors of GIB in our patients prescribed with dabigatran included age $\geq 75$, history of peptic ulcer or GIB, and concomitant use of aspirin.

Gastroprotective agents were associated with a reduced risk of GIB in patients on dabigatran. The protective effect seemed to be more on prevention of upper GIB and among those with a prior history of peptic ulcers or GIB. Hence, gastroprotective agents shall be considered in highrisk patients taking dabigatran. Further prospective randomized clinical trial is warranted to confirm the effect of gastroprotective agents on reducing the risk of GIB in patients using dabigatran.

\section{Acknowledgement}

We thank our colleagues in the Department of Pharmacology and Pharmacy of the University of Hong Kong - Ms. Celine SL Chui, for her contribution to initial data retrieval; Mr. Kenneth KC Man, for cross-checking the statistical analysis; and Mrs. Lisa Wong, for proofreading the manuscript. 


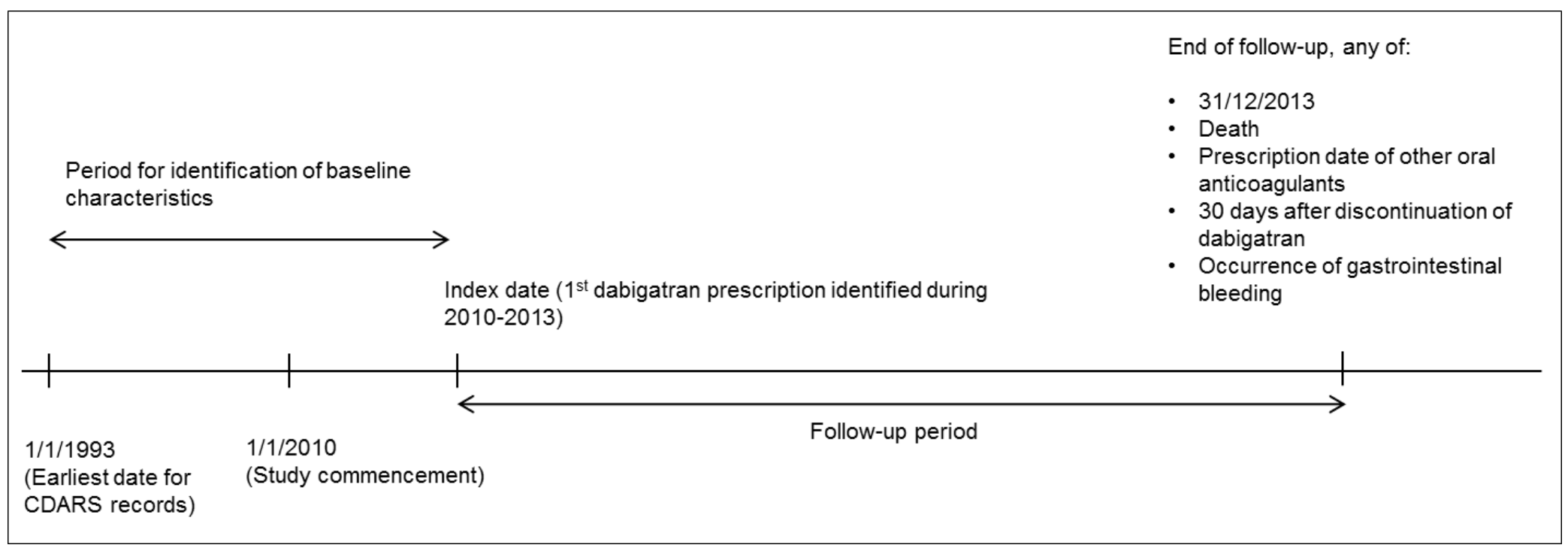

Figure 1. Study design 


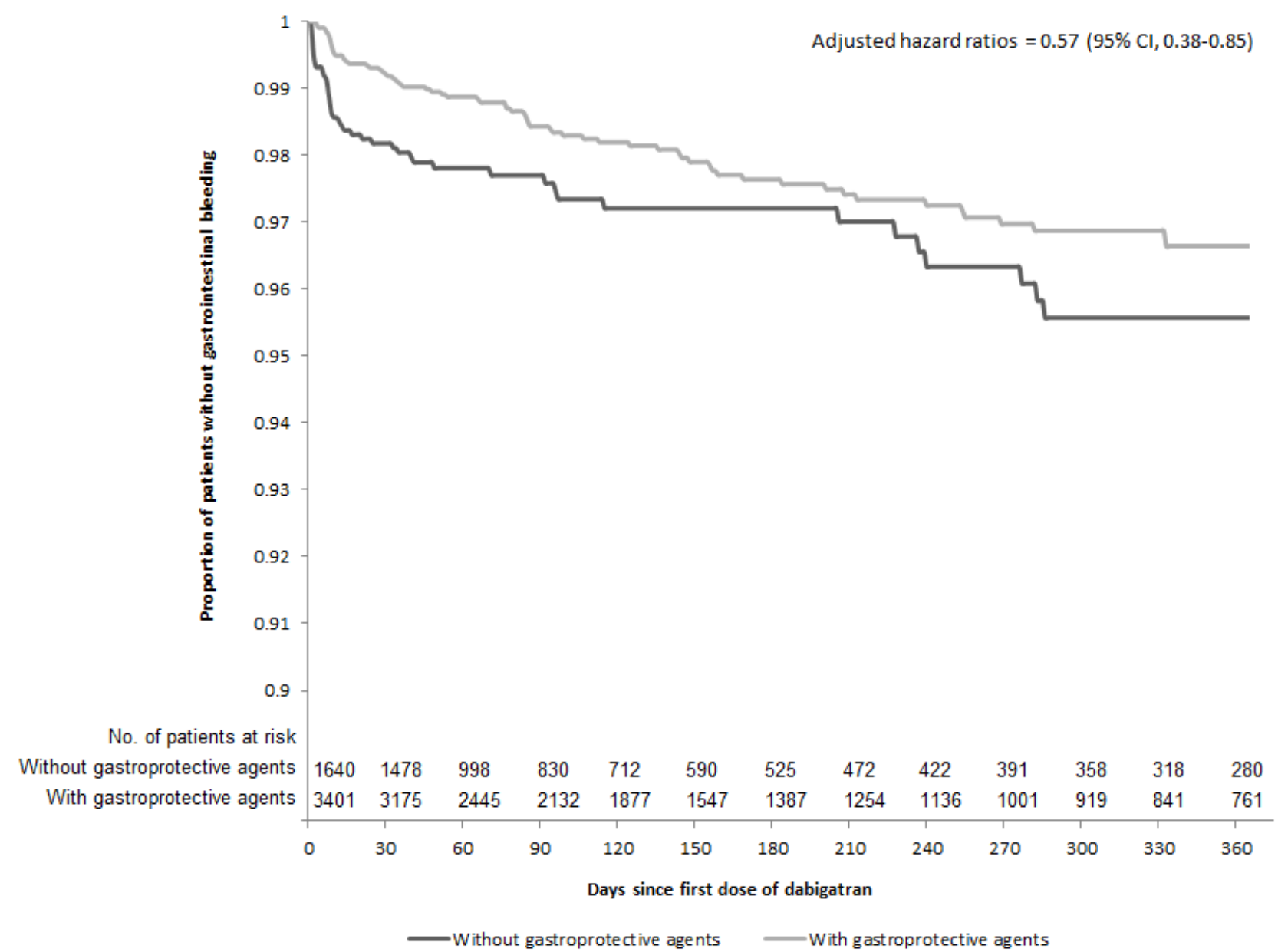

Figure 2. Kaplan-Meier curves of gastrointestinal bleeding. CI, confidence interval. 
Table 1. Baseline characteristics

\begin{tabular}{|c|c|}
\hline Characteristics & $\mathrm{n}(\%)$ \\
\hline Total & 5041 \\
\hline Age, mean \pm standard deviation & $72.0 \pm 10.9$ \\
\hline Age $\geq 75$ & $2368(47.0)$ \\
\hline Sex (Male) & $2412(47.8)$ \\
\hline \multicolumn{2}{|l|}{ Baseline medical conditions } \\
\hline Congestive heart failure & $893(17.7)$ \\
\hline Hypertension & $2377(47.2)$ \\
\hline Diabetes mellitus & $1079(21.4)$ \\
\hline Prior ischemic stroke/TIA/SE & $1413(28.0)$ \\
\hline Renal disease & $257(5.1)$ \\
\hline History of myocardial infarction & $209(4.1)$ \\
\hline History of intracranial bleeding & $121(2.4)$ \\
\hline History of peptic ulcer/GIB & $700(13.9)$ \\
\hline \multicolumn{2}{|l|}{ Concurrent medications } \\
\hline Aspirin & $1538(30.5)$ \\
\hline Clopidogrel & $211(4.2)$ \\
\hline Gastroprotective agents ${ }^{\mathrm{a}}$ & $3401(67.5)$ \\
\hline NSAIDs & $523(10.4)$ \\
\hline SSRIs & $210(4.2)$ \\
\hline \multicolumn{2}{|l|}{ Indication for dabigatran } \\
\hline Atrial fibrillation & $4451(88.3)$ \\
\hline Prophylaxis of VTE & $572(11.3)$ \\
\hline Non-specified & $18(0.4)$ \\
\hline \multicolumn{2}{|c|}{ Dosage of dabigatran (average daily dose) } \\
\hline$<220 \mathrm{mg}$ & $502(10.0)$ \\
\hline $220 \mathrm{mg}$ & $3686(73.1)$ \\
\hline$>220 \mathrm{mg}$ & $730(14.5)$ \\
\hline Non-specified & $123(2.4)$ \\
\hline
\end{tabular}

Abbreviations: TIA, transient ischemic attack; SE, systemic embolism; GIB, gastrointestinal bleeding; NSAIDs, nonsteroidal anti-inflammatory drugs; SSRIs, selective serotonin reuptake inhibitors; VTE, venous thromboembolism.

${ }^{a}$ include proton pump inhibitors and histamine type- 2 receptor antagonists 
Table 2. Results of the main analysis

\begin{tabular}{lcc}
\hline & $\mathrm{n}(\%)$ & Incidence rate ratios $(95 \% \mathrm{CI})$ \\
\hline Total no. of patients & 5041 & - \\
Total no. of patient years & 2973 & - \\
No. of GIB & $124(2.5)$ & - \\
Age $\geq 75$ & $2368(47.0)$ & $2.47(1.66,3.68)^{*}$ \\
Sex (Male) & $2412(47.8)$ & $0.80(0.55,1.14)$ \\
Baseline conditions & & \\
Prior ischemic stroke/TIA/SE & $1413(28.0)$ & $1.31(0.91,1.89)$ \\
Renal disease & $257(5.1)$ & $0.63(0.26,1.56)$ \\
History of peptic ulcer/GIB & $700(13.9)$ & $2.31(1.54,3.46)^{*}$ \\
Concurrent Medication & & \\
Aspirin & $1538(30.5)$ & $1.52(1.03,2.24)^{*}$ \\
Clopidogrel & $211(4.2)$ & $0.37(0.09,1.51)$ \\
Gastroprotective agents & $3401(67.5)$ & $0.52(0.35,0.77)^{*}$ \\
$\quad$ Histamine type-2 receptor antagonists & $2004(39.8)$ & $0.61(0.40,0.94)^{*}$ \\
$\quad$ Proton pump inhibitors & $923(18.3)$ & $0.53(0.31,0.91)^{*}$ \\
$\quad$ Both & $474(9.4)$ & $0.15(0.06,0.39)^{*}$ \\
NSAIDs & $523(10.4)$ & $0.51(0.22,1.17)$ \\
SSRIs & $210(4.2)$ & $0.51(0.19,1.40)$ \\
\hline Abbrevith & & \\
\hline
\end{tabular}

Abbreviations: GIB, gastrointestinal bleeding; TIA, transient ischemic attack; SE, systemic embolism; NSAIDs, nonsteroidal anti-inflammatory drugs; SSRIs, selective serotonin reuptake inhibitors; $* \mathrm{p}<0.05$. 
Table 3. Sensitivity analyses of the effect of gastroprotective agents on gastrointestinal bleeding

\begin{tabular}{|c|c|c|c|c|}
\hline & $\mathrm{N}$ & No. of GIB (\%) & Incidence rate ratio $(95 \% \mathrm{CI})$ & p-value ${ }^{a}$ \\
\hline Main model & 5041 & $124(2.5)$ & $0.52(0.35,0.77)$ & \\
\hline \multicolumn{5}{|l|}{ Stratified by prior OAC experience ${ }^{b}$} \\
\hline New OAC starters & 3967 & $91(2.3)$ & $0.56(0.35,0.89)$ & 0.40 \\
\hline Switchers & 1074 & $33(3.1)$ & $0.38(0.18,0.82)$ & \\
\hline \multicolumn{5}{|l|}{ Subcategory analyses of the source of GIB } \\
\hline Upper GIB & 5041 & $46(0.9)$ & $0.29(0.15,0.54)$ & 0.02 \\
\hline Non-upper GIB & 5041 & $78(1.5)$ & $0.75(0.45,1.27)$ & \\
\hline \multicolumn{5}{|l|}{ Stratified by history of peptic ulcer/GIB } \\
\hline With history peptic ulcer/GIB & 700 & $34(4.9)$ & $0.14(0.06,0.30)$ & $<0.001$ \\
\hline No history of peptic ulcer/GIB & 4341 & $90(2.1)$ & $0.80(0.50,1.28)$ & \\
\hline Restricted to patients with atrial fibrillation only & 4451 & $119(2.7)$ & $0.58(0.39,0.87)$ & \\
\hline Adjusted for dosage effect of dabigatran & $4918^{c}$ & $120(2.4)$ & $0.53(0.36,0.80)$ & \\
\hline Early bleeding of dabigatran ${ }^{\mathrm{d}}$ & 5041 & $56(1.1)$ & $0.39(0.22,0.69)$ & \\
\hline
\end{tabular}

Abbreviations: GIB, gastrointestinal bleeding; OAC, oral anticoagulant.

${ }^{a}$ t-test was used to test for any differences in effect estimates within sub-groups.

${ }^{\mathrm{b}}$ Patients were classified as switchers if they were prescribed warfarin, rivaroxaban, or apixaban within 3 months prior to index date.

Otherwise they were classified as new OAC starters.

c $123(2 \%)$ patients were excluded in the analysis due to unspecified dosage.

${ }^{\mathrm{d}}$ GIB that occurred within 30 days since commencement of dabigatran. 
Table 4. Stratification into different use of gastroprotective agents

\begin{tabular}{|c|c|c|c|c|}
\hline & Overall $^{\mathrm{a}}$ & H2RA alone & PPI alone & $\begin{array}{c}\text { Both H2RA and } \\
\text { PPI }^{\mathrm{b}}\end{array}$ \\
\hline All users, n (\%) & 3,401 & 2,004 & 923 & 474 \\
\hline Continuous users $^{\mathrm{c}}, \mathrm{n}(\%)$ & $2,423(71.2)$ & $1,393(69.5)$ & $660(71.5)$ & $370(78.1)$ \\
\hline Infrequent users ${ }^{\mathrm{c}}, \mathrm{n}(\%)$ & $978(28.8)$ & $611(30.5)$ & $263(28.5)$ & $104(21.9)$ \\
\hline \multicolumn{5}{|c|}{ Main model, incidence rate ratios (95\% CI) } \\
\hline All users & $0.52(0.35,0.77)$ & $0.61(0.40,0.94)$ & $0.53(0.31,0.91)$ & $0.15(0.06,0.39)$ \\
\hline Continuous users $^{c}$ & $0.51(0.34,0.77)$ & $0.62(0.40,0.97)$ & $0.48(0.26,0.87)$ & $0.18(0.07,0.47)$ \\
\hline Infrequent users ${ }^{c}$ & $0.54(0.31,0.96)$ & $0.59(0.30,1.15)$ & $0.76(0.32,1.79)$ & $-{ }^{d}$ \\
\hline \multicolumn{5}{|c|}{ Time-to-event analysis, hazard rate ratios $(95 \% \mathrm{CI})$} \\
\hline All users & $0.57(0.38,0.85)$ & $0.66(0.43,1.01)$ & $0.57(0.34,0.97)$ & $0.19(0.07,0.49)$ \\
\hline Continuous users ${ }^{\mathrm{c}}$ & $0.57(0.38,0.87)$ & $0.68(0.43,1.06)$ & $0.53(0.29,0.95)$ & $0.23(0.09,0.60)$ \\
\hline Infrequent users ${ }^{c}$ & $0.57(0.32,1.00)$ & $0.61(0.31,1.19)$ & $0.74(0.32,1.75)$ & $-{ }^{d}$ \\
\hline
\end{tabular}

Abbreviations: H2RA, histamine type-2 receptor antagonists; PPI, proton pump inhibitors; CI, confidence interval.

a Includes H2RA and/or PPI.

${ }^{\mathrm{b}}$ Includes H2RA and PPI, either prescribed simultaneously or sequentially.

${ }^{\mathrm{c}}$ Patients who had day supply of drugs with $\geq 80 \%$ of the days of follow-up were classified as continuous users; otherwise they were classified as infrequent users.

${ }^{\mathrm{d}}$ No patients in this group developed gastrointestinal bleeding. 


\section{References}

1. Hankey GJ, Eikelboom JW. Dabigatran etexilate: a new oral thrombin inhibitor. Circulation. 2011;123(13):1436-1450.

2. Connolly SJ, Ezekowitz MD, Yusuf S, et al. Dabigatran versus warfarin in patients with atrial fibrillation. N Engl J Med. 2009;361(12):1139-1151.

3. Schulman S, Kearon C, Kakkar AK, et al. Dabigatran versus Warfarin in the Treatment of Acute Venous Thromboembolism. N Engl J Med. 2009;361(24):2342-2352.

4. Oldgren J, Budaj A, Granger CB, et al. Dabigatran vs. placebo in patients with acute coronary syndromes on dual antiplatelet therapy: a randomized, double-blind, phase II trial. Eur Heart J. 2011;32(22):2781-2789.

5. McConeghy KW, Bress A, Qato DM, et al. Evaluation of Dabigatran Bleeding Adverse Reaction Reports in the FDA Adverse Event Reporting System during the First Year of Approval. Pharmacotherapy. 2014. doi: 10.1002/phar.1415.

6. Holster IL, Valkhoff VE, Kuipers EJ, et al. New oral anticoagulants increase risk for gastrointestinal bleeding: a systematic review and meta-analysis. Gastroenterology. 2013;145(1):105-112.

7. The U.S. Food and Drug Administration (FDA). FDA study of Medicare patients finds risks lower for stroke and death but higher for gastrointestinal bleeding with Pradaxa (dabigatran) compared to warfarin. Available at: http://www.fda.gov/Drugs/DrugSafety/ucm396470.htm. Accessed November 27, 2014.

8. Heidbuchel H, Verhamme P, Alings M, et al. European Heart Rhythm Association Practical Guide on the use of new oral anticoagulants in patients with non-valvular atrial fibrillation. Europace. 2013;15(5):625-651.

9. Abraham NS, Hlatky MA, Antman EM, et al. ACCF/ACG/AHA 2010 Expert Consensus Document on the concomitant use of proton pump inhibitors and thienopyridines: a focused update of the ACCF/ACG/AHA 2008 expert consensus document on reducing the gastrointestinal risks of antiplatelet therapy and NSAID use: a report of the American College of Cardiology Foundation Task Force on Expert Consensus Documents. Circulation. 2010;122(24):2619-2633.

10. Agewall S, Cattaneo M, Collet JP, et al. Expert position paper on the use of proton pump inhibitors in patients with cardiovascular disease and antithrombotic therapy. Eur Heart J. 2013;34(23):1708-1715.

11. The Hospital Authority. Introduction. Available at: http://www.ha.org.hk/visitor/ha visitor text index.asp?Content ID=10008\&Lang=ENG\&Dimen sion=100\&Parent_ID=10004\&Ver=TEXT. Accessed November 27, 2014.

12. CP Wong. Health Informatics Development in the Hospital Authority. Available at: http://www.ha.org.hk/haconvention/hac2006proceedings/doc/S10_2.pdf. Accessed March 22, 2015.

13. Man KK, Ip P, Hsia Y, et al. ADHD Drug Prescribing Trend Is Increasing Among Children and Adolescents in Hong Kong. J Atten Disord. 2014. doi: 10.1177/1087054714536047.

14. Chiu SS, Lau YL, Chan KH, et al. Influenza-related hospitalizations among children in Hong Kong. N Engl J Med. 2002;347(26):2097-2103.

15. He Y, Chan EW, Man KK, et al. Dosage Effects of Histamine-2 Receptor Antagonist on the Primary Prophylaxis of Non-Steroidal Anti-Inflammatory Drug (NSAID)-Associated Peptic Ulcers: A Retrospective Cohort Study. Drug Saf. 2014.

16. Chui CS, Man KK, Cheng CL, et al. An investigation of the potential association between retinal detachment and oral fluoroquinolones: a self-controlled case series study. J Antimicrob Chemother. 2014. doi: 10.1093/jac/dku145. 
17. Man KK, Chan EW, Coghill D, et al. Methylphenidate and the risk of trauma. Pediatrics. 2015;135(1):40-48.

18. Wong OF, Ho PL, Lam SK. Retrospective review of clinical presentations, microbiology, and outcomes of patients with psoas abscess. Hong Kong Med J. 2013;19(5):416-423.

19. Masclee GMC, Valkhoff VE, Coloma PM, et al. Risk of Upper Gastrointestinal Bleeding From Different Drug Combinations. Gastroenterology.147(4):784-792.e789.

20. European Medicines Agency. CHMP assessment report: Pradaxa. Procedure No. EMEA/H/C/000829/X/13/G. Available at: www.ema.europa.eu/docs/en_GB/document_library/EPAR_-_Assessment_Report_Variation/human/000829/WC500110875.pdf. Accessed November 27, 2014.

21. Eikelboom JW, Wallentin L, Connolly SJ, et al. Risk of bleeding with 2 doses of dabigatran compared with warfarin in older and younger patients with atrial fibrillation: an analysis of the randomized evaluation of long-term anticoagulant therapy (RE-LY) trial. Circulation. 2011;123(21):2363-2372.

22. Desai J, Kolb JM, Weitz JI, et al. Gastrointestinal bleeding with the new oral anticoagulants-defining the issues and the management strategies. Thromb Haemost. 2013;110(2):205-212.

23. McDonald CJ, Kalisch Ellett LM, Barratt JD, et al. An international comparison of spontaneous adverse event reports and potentially inappropriate medicine use associated with dabigatran. Pharmacoepidemiol Drug Saf. 2014.

24. Hospital Authority. Clinical Services Plan for the Hong Kong West Cluster. Available at: http://www.ha.org.hk/upload/publication_44/453.pdf.

25. Census and Statistics Department HKSAR. Population and Household Statistics Analysed by District Council District 2013. Available at: http://www.statistics.gov.hk/pub/B11303012013AN13B0100.pdf. Accessed March 22, 2015.

26. Sorensen R, Gislason G, Torp-Pedersen C, et al. Dabigatran use in Danish atrial fibrillation patients in 2011: a nationwide study. BMJ Open. 2013;3(5).

27. Kernan L, Ito S, Shirazi F, et al. Fatal gastrointestinal hemorrhage after a single dose of dabigatran. Clin Toxicol (Phila). 2012;50(7):571-573.

28. Stollberger C, Lindner K, Finsterer J. Gastrointestinal bleeding under dabigatran. J Postgrad Med. 2014;60(2):192-193.

29. Carter A, Sarda P, George M, et al. Hip arthroplasty fatality related to dabigatran induced gastrointestinal haemorrhage. Ann R Coll Surg Engl. 2014;96(1):115E-117E.

30. Fellows SE, Rosini JM, Curtis JA, et al. Hemorrhagic Gastritis with Dabigatran in a Patient with Renal Insufficiency. J Emerg Med. 2013;44(2):e221-e225.

31. The Hospital Authority. Hospital Authority Statistical Report 2012 - 2013. Available at: http://www.ha.org.hk/upload/publication_15/491.pdf. Accessed November 27, 2014.

32. Lanza FL, Chan FK, Quigley EM. Guidelines for prevention of NSAID-related ulcer complications. Am J Gastroenterol. 2009;104(3):728-738.

33. Valkhoff VE, Sturkenboom MCJM, Kuipers EJ. Risk factors for gastrointestinal bleeding associated with low-dose aspirin. Best Pract Res Cl Ga. 2012;26(2):125-140.

34. Hernandez-Diaz S, Rodriguez LAG. Association between nonsteroidal anti-inflammatory drugs and upper gastrointestinal tract bleeding/perforation - An overview of epidemiologic studies published in the 1990s. Arch Intern Med. 2000;160(14):2093-2099.

35. Joppi R, Cinconze E, Mezzalira L, et al. Hospitalized patients with atrial fibrillation compared to those included in recent trials on novel oral anticoagulants: A population-based study. Eur J Intern Med. 2013;24(4):318-323.

36. Eric J Stanek, Barnabie C Agatep, Vivian Herrera, et al. Abstract 16675: Assessment of Initial U.S. Dabigatran Utilization and Short-Term Persistence. Session Title: Epidemiology of Atrial Fibrillation. Circulation. 2011;124(A16675). 
37. Larsen TB, Rasmussen LH, Skjoth F, et al. Efficacy and safety of dabigatran etexilate and warfarin in "real-world" patients with atrial fibrillation: a prospective nationwide cohort study. J Am Coll Cardiol. 2013;61(22):2264-2273.

38. Sung JJ, Kuipers EJ, El-Serag HB. Systematic review: the global incidence and prevalence of peptic ulcer disease. Aliment Pharmacol Ther. 2009;29(9):938-946.

39. Woo J, Ho SC, Chan SG, et al. An estimate of chronic disease burden and some economic consequences among the elderly Hong Kong population. J Epidemiol Commun $\mathrm{H}$. 1997;51(5):486-489.

40. Leong RW. Differences in Peptic Ulcer Between the East and the West. Gastroenterol Clin N. 2009;38(2):363-379.

41. WHO and Department of Health Hong Kong. Health Service Delivery Profile. Hong Kong (China). 2012. Available at:

http://www.wpro.who.int/health services/service delivery_profile hong kong \%28china\%29.pd f. Accessed January 30, 2015.

42. The U.S. Food and Drug Administration (FDA). Briefing Information, Dabigatran Etexilate Mesylate Capsules, for the September 20, 2010 Meeting of the Cardiovascular and Renal Drugs Advisory Committee. Available at:

http://www.fda.gov/downloads/AdvisoryCommittees/CommitteesMeeting\%20Materials/Drugs/C ardiovascularandRenalDrugsAdvisoryCommittee/UCM247244. Accessed November 27, 2014.

43. Hennessy S. Use of health care databases in pharmacoepidemiology. Basic Clin Pharmacol Toxicol. 2006;98(3):311-313.

44. Takahashi Y, Nishida Y, Asai S. Utilization of health care databases for pharmacoepidemiology. Eur J Clin Pharmacol. 2012;68(2):123-129.

45. Wong IC, Murray ML. The potential of UK clinical databases in enhancing paediatric medication research. Br J Clin Pharmacol. 2005;59(6):750-755.

46. Black N. Why we need observational studies to evaluate the effectiveness of health care. BMJ. 1996;312(7040):1215-1218.

47. Southworth MR, Reichman ME, Unger EF. Dabigatran and Postmarketing Reports of Bleeding. N Engl J Med. 2013;368(14):1272-1274.

48. Hori M, Connolly SJ, Zhu J, et al. Dabigatran versus warfarin: effects on ischemic and hemorrhagic strokes and bleeding in Asians and non-Asians with atrial fibrillation. Stroke. 2013;44(7):1891-1896.

49. Shen AY, Yao JF, Brar SS, et al. Racial/ethnic differences in the risk of intracranial hemorrhage among patients with atrial fibrillation. J Am Coll Cardiol. 2007;50(4):309-315.

50. Gregg JP, Yamane AJ, Grody WW. Prevalence of the factor V-Leiden mutation in four distinct American ethnic populations. Am J Med Genet. 1997;73(3):334-336.

51. Kujovich JL. Factor V Leiden thrombophilia. Genet Med. 2011;13(1):1-16.

52. van Asch CJJ, Luitse MJA, Rinkel GJE, et al. Incidence, case fatality, and functional outcome of intracerebral haemorrhage over time, according to age, sex, and ethnic origin: a systematic review and meta-analysis. Lancet Neurol. 2010;9(2):167-176.

53. Yasaka M, Lip GY. Impact of non-vitamin k antagonist oral anticoagulants on intracranial bleeding in Asian patients with non-valvular atrial fibrillation. Circ J. 2014;78(10):2367-2372.

54. Drug Office, Department of Health, The Government of the Hong Kong Special Administrative Region. Registered Pharmaceutical Products. Available at: http://www.drugoffice.gov.hk/eps/do/en/consumer/home.html. Accessed February 2, 2015.

55. Hernandez I, Baik S, Piñera A, et al. Risk of bleeding with dabigatran in atrial fibrillation. JAMA Intern Med. 2014. 
56. Graham DJ, Reichman ME, Wernecke M, et al. Cardiovascular, Bleeding, and Mortality Risks in Elderly Medicare Patients Treated with Dabigatran or Warfarin for Non-Valvular Atrial Fibrillation. Circulation. 2014.

57. Vaughan Sarrazin MS, Jones M, Mazur A, et al. Bleeding rates in Veterans Affairs patients with atrial fibrillation who switch from warfarin to dabigatran. Am J Med. 2014.

Author names in bold designate shared co-first authors. 
Supplementary materials

Supplementary Figure 1. Data validation

Supplementary Table 1. International Classification of Diseases, Ninth Revision, Clinical Modification (ICD-9) diagnosis codes used in this study 


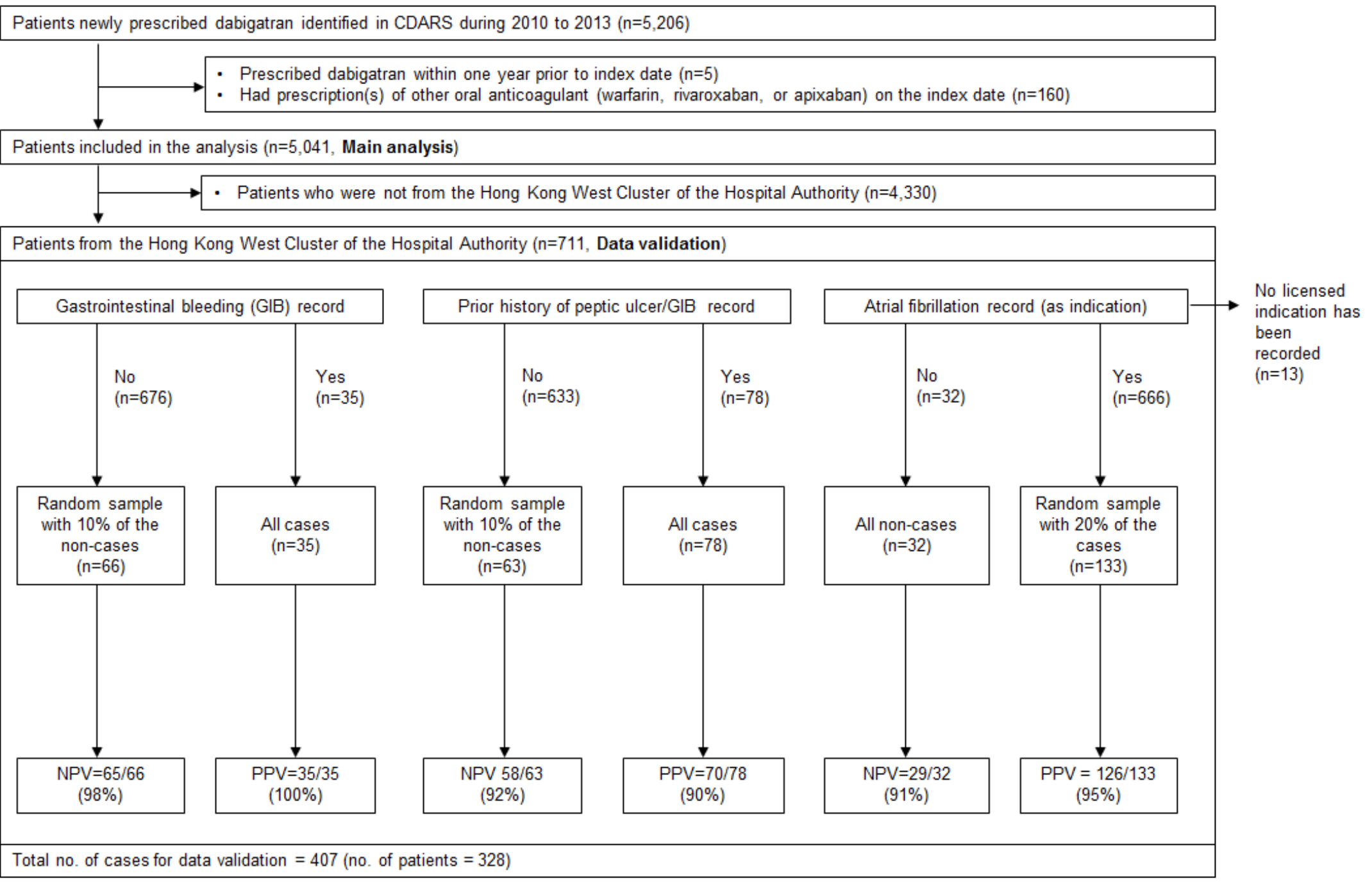

\section{Supplementary Figure 1. Data validation}




\section{Supplementary Table 1. International Classification of Diseases, Ninth Revision, Clinical Modification (ICD-9) diagnosis codes used in this study}

\begin{tabular}{|c|c|}
\hline ICD-9 codes & Descriptions \\
\hline \multicolumn{2}{|c|}{ Gastrointestinal bleeding (GIB) } \\
\hline \multicolumn{2}{|c|}{ Upper GIB } \\
\hline 531.0 & Acute gastric ulcer with hemorrhage \\
\hline 531.2 & Acute gastric ulcer with hemorrhage and perforation, without mention of obstruction \\
\hline 531.4 & Chronic or unspecified gastric ulcer with hemorrhage \\
\hline 531.6 & Chronic or unspecified gastric ulcer with hemorrhage and perforation \\
\hline 532.0 & Acute duodenal ulcer with hemorrhage \\
\hline 532.2 & Acute duodenal ulcer with hemorrhage and perforation \\
\hline 532.4 & Chronic or unspecified duodenal ulcer with hemorrhage \\
\hline 532.6 & Chronic or unspecified duodenal ulcer with hemorrhage and perforation \\
\hline 533.0 & Acute peptic ulcer of unspecified site with hemorrhage \\
\hline 533.2 & Acute peptic ulcer of unspecified site with hemorrhage and perforation \\
\hline 533.4 & Chronic or unspecified peptic ulcer of unspecified site with hemorrhage \\
\hline 533.6 & $\begin{array}{l}\text { Chronic or unspecified peptic ulcer of unspecified site with hemorrhage and } \\
\text { perforation }\end{array}$ \\
\hline 534.0 & Acute gastrojejunal ulcer with hemorrhage \\
\hline 534.2 & $\begin{array}{l}\text { Acute gastrojejunal ulcer with hemorrhage and perforation, without mention of } \\
\text { obstruction }\end{array}$ \\
\hline 534.4 & Chronic or unspecified gastrojejunal ulcer with hemorrhage \\
\hline 534.6 & Chronic or unspecified gastrojejunal ulcer with hemorrhage and perforation \\
\hline 535.01 & Acute gastritis, with hemorrhage \\
\hline 535.11 & Atrophic gastritis, with hemorrhage \\
\hline 535.21 & Gastric mucosal hypertrophy, with hemorrhage \\
\hline 535.31 & Alcoholic gastritis, with hemorrhage \\
\hline 535.41 & Other specified gastritis, with hemorrhage \\
\hline 535.51 & Unspecified gastritis and gastroduodenitis, with hemorrhage \\
\hline 535.61 & Duodenitis, with hemorrhage \\
\hline 535.71 & Eosinophilic gastritis, with hemorrhage \\
\hline 578.0 & Hematemesis \\
\hline 578.1 & Melena \\
\hline $578.9^{*}$ & Hemorrhage of gastrointestinal tract, unspecified \\
\hline \multicolumn{2}{|l|}{ Other GIB } \\
\hline 562.02 & Diverticulosis of small intestine with hemorrhage \\
\hline 562.03 & Diverticulitis of small intestine with haemorrhage \\
\hline 562.12 & Diverticulosis of colon with haemorrhage \\
\hline 562.13 & Diverticulitis of colon with haemorrhage \\
\hline 569.3 & Hemorrhage of rectum and anus \\
\hline 569.85 & Angiodysplasia of intestine with haemorrhage \\
\hline 569.86 & Dieulafoy lesion (hemorrhagic) of intestine \\
\hline
\end{tabular}




\section{Supplementary Table 1. International Classification of Diseases, Ninth Revision, Clinical Modification (ICD-9) diagnosis codes used in this study (continued).}

\begin{tabular}{|c|c|}
\hline ICD-9 codes & Descriptions \\
\hline \multicolumn{2}{|c|}{ Congestive Heart Failure } \\
\hline 398.91 & Rheumatic heart failure (congestive) \\
\hline 402.01 & Malignant hypertensive heart disease with heart failure \\
\hline 402.11 & Benign hypertensive heart disease with heart failure \\
\hline 402.91 & Unspecified hypertensive heart disease with heart failure \\
\hline 404.01 & $\begin{array}{l}\text { Hypertensive heart and chronic kidney disease, malignant, with heart failure and } \\
\text { with chronic kidney disease stage I through stage IV, or unspecified }\end{array}$ \\
\hline 404.03 & $\begin{array}{l}\text { Hypertensive heart and chronic kidney disease, malignant, with heart failure and } \\
\text { with chronic kidney disease stage } \mathrm{V} \text { or end stage renal disease }\end{array}$ \\
\hline 404.11 & $\begin{array}{l}\text { Hypertensive heart and chronic kidney disease, benign, with heart failure and with } \\
\text { chronic kidney disease stage I through stage IV, or unspecified }\end{array}$ \\
\hline 404.13 & $\begin{array}{l}\text { Hypertensive heart and chronic kidney disease, benign, with heart failure and } \\
\text { chronic kidney disease stage } \mathrm{V} \text { or end stage renal disease }\end{array}$ \\
\hline 404.91 & $\begin{array}{l}\text { Hypertensive heart and chronic kidney disease, unspecified, with heart failure and } \\
\text { with chronic kidney disease stage I through stage IV, or unspecified }\end{array}$ \\
\hline 404.93 & $\begin{array}{l}\text { Hypertensive heart and chronic kidney disease, unspecified, with heart failure and } \\
\text { chronic kidney disease stage } \mathrm{V} \text { or end stage renal disease }\end{array}$ \\
\hline 428 & Heart failure \\
\hline \multicolumn{2}{|l|}{ Hypertension } \\
\hline 401 & Essential hypertension \\
\hline 402 & Hypertensive heart disease \\
\hline 403 & Hypertensive chronic kidney disease \\
\hline 404 & Hypertensive heart and chronic kidney disease \\
\hline 405 & Secondary hypertension \\
\hline 437.2 & Hypertensive encephalopathy \\
\hline \multicolumn{2}{|l|}{ Diabetes } \\
\hline 250 & Diabetes mellitus \\
\hline \multicolumn{2}{|c|}{ Ischemic stroke/transient ischemic attack/systemic embolism } \\
\hline 433.01 & Occlusion and stenosis of basilar artery with cerebral infarction \\
\hline 433.11 & Occlusion and stenosis of carotid artery with cerebral infarction \\
\hline 433.21 & Occlusion and stenosis of vertebral artery with cerebral infarction \\
\hline 433.31 & $\begin{array}{l}\text { Occlusion and stenosis of multiple and bilateral precerebral arteries with cerebral } \\
\text { infarction }\end{array}$ \\
\hline 433.81 & Occlusion and stenosis of other specified precerebral artery with cerebral infarction \\
\hline 433.91 & Occlusion and stenosis of unspecified precerebral artery with cerebral infarction \\
\hline 434 & Occlusion of cerebral arteries \\
\hline
\end{tabular}




\section{Supplementary Table 1. International Classification of Diseases, Ninth Revision, Clinical Modification (ICD-9) diagnosis codes used in this study (continued).}

\begin{tabular}{|c|c|}
\hline ICD-9 codes & Descriptions \\
\hline \multicolumn{2}{|c|}{ Ischemic stroke/transient ischemic attack/systemic embolism (continued) } \\
\hline 435 & Transient cerebral ischemia \\
\hline 436 & Acute, but ill-defined, cerebrovascular disease \\
\hline 437.0 & Cerebral atherosclerosis \\
\hline 437.1 & Other generalized ischemic cerebrovascular disease \\
\hline 444 & Arterial embolism and thrombosis \\
\hline 445 & Atheroembolism \\
\hline \multicolumn{2}{|c|}{ Renal disease } \\
\hline 403 & Hypertensive chronic kidney disease \\
\hline 404 & Hypertensive heart and chronic kidney disease \\
\hline 580 & Acute glomerulonephritis \\
\hline 581 & Nephrotic syndrome \\
\hline 582 & Chronic glomerulonephritis \\
\hline 583 & Nephritis and nephropathy not specified as acute or chronic \\
\hline 584 & Acute kidney failure \\
\hline 585 & Chronic kidney disease (ckd) \\
\hline 586 & Renal failure unspecified \\
\hline 590.0 & Chronic pyelonephritis \\
\hline 753.1 & Cystic kidney disease \\
\hline \multicolumn{2}{|c|}{ Myocardial infarction } \\
\hline 410 & Acute myocardial infarction \\
\hline \multicolumn{2}{|c|}{ Intracranial hemorrhage } \\
\hline 430 & Subarachnoid hemorrhage \\
\hline 431 & Intracerebral hemorrhage \\
\hline 432 & Other and unspecified intracranial hemorrhage \\
\hline \multicolumn{2}{|c|}{ Peptic ulcer/ Gastrointestinal bleeding } \\
\hline 531 & Gastric ulcer \\
\hline 532 & Duodenal ulcer \\
\hline 533 & Peptic ulcer, site unspecified \\
\hline 534 & Gastrojejunal ulcer \\
\hline 535 & Gastritis and duodenitis \\
\hline 578 & Gastrointestinal hemorrhage \\
\hline \multicolumn{2}{|c|}{ Atrial fibrillation } \\
\hline 427.3 & Atrial fibrillation and flutter \\
\hline
\end{tabular}

